\section{ÍNDICE TORNOZELO-BRAÇO NOS PACIENTES SUBMETIDOS À PROGRAMA DE EXERCÍCIO SUPERVISIONADO}

\author{
INDEX ANKLE-ARM IN PATIENTS SUBMITTED TO SUPERVISED EXERCISE PROGRAM \\ ÍNDICE TOBILLO-BRAZO EN PACIENTES SOMETIDOS A PROGRAMA DE EJERCICIO SUPERVISADO
}

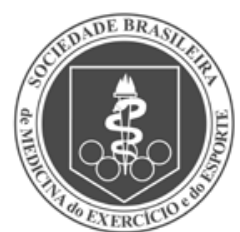

Artigo Original

ORIGINAL ARTICLE

Artículo Original
Marco Antonio Alves Azizi' (Médico)

1. Universidade Iguaçu, Nova

Iguaçu, RJ, Brasil.

\section{Correspondência:}

Estrada Padre Roser 223, Vila da Penha Rio de Janeiro, RJ, Brasil. 21220-560. marcoazizi@oi.com.br

\section{RESUMO}

Introdução: o índice tornozelo-braço (ITB) é o primeiro, o mais simples e o mais importante teste não invasivo para detecção de doença arterial obstrutiva periférica (DAOP). Objetivo: medir a integridade da circulação arterial dos membros inferiores. Avaliar e correlacionar o ITB com a realização do programa de exercício supervisionado (PES) em indivíduos que apresentaram as principais indicações para o exame, levando-se em conta o perfil de risco da população. Método: vinte e cinco pacientes participantes do PES com indicação de ITB, com média de idade de 66,6 anos (52-83), todos frequentando o PES há, no mínimo, seis meses. Foi medida a pressão sistólica no membro superior e a pressão sistólica nos tornozelos antes e após a realização do PES em cicloergômetro de membros inferiores ou esteira rolante. O ITB foi calculado para cada membro inferior utilizando-se o maior valor como numerador e o maior valor encontrado nas medidas braquiais como denominador. Resultados: após a realização do exame, os resultados obtidos foram variados com aumento, diminuição e manutenção dos valores do ITB independentemente de idade, sexo e patologias presentes nos pacientes analisados. Conclusão: nenhum paciente obteve ITB $\leq 0,90$, tanto no pré como no pós-esforço. 0 exercício mostrou-se benéfico para a irrigação dos membros inferiores.

Palavras-chave: índice tornozelo-braço, terapia por exercício, esforço físico.

\begin{abstract}
Introduction: the ankle-brachial index $(A B I)$ is the first, the simplest and the most important non-invasive test for the detection of peripheral arterial disease (PAD). Objective: to measure the integrity of the arterial circulation of the lower limbs. Evaluate and correlate ABI with the completion of supervised exercise program (SEP) in individuals who presented the main indications for the examination, taking into account the risk profile of the population. Method: twenty-five patients participating in the SEP with indications of ITB, with a mean age of 66.6 years (52-83), all attending the SEP for at least six months. The systolic pressure was measured in the arm and systolic blood pressure in the ankles before and after the completion of the SEP in ergometer cycle for lower limbs or in a treadmill. The ABI was calculated for each leg and the highest was used as the numerator, and the highest value found in the brachial measures as the denominator. Results: after the examination the results were varied to increase, decrease and maintain the ABI values regardless of age, sex and diseases present in the patients studied. Conclusion: no patient had $A B I \leq 0.90$, both pre and post-exercise. The exercise proved to be beneficial for the irrigation of the lower limbs.
\end{abstract}

Keywords: ankle brachial index, exercise therapy, physical exertion.

\section{RESUMEN}

Introducción: el índice tobillo-brazo (ITB) es la primera, la más simple y la más importante prueba no invasiva para la detección de la enfermedad arterial periférica (EAP). Objetivo: medir la integridad de la circulación arterial de las extremidades inferiores. Evaluar y correlacionar el índice tobillo-brazo (ITB) con la realización de un programa de ejercicio supervisado (PES) en los individuos que presentaron las principales indicaciones para el examen, teniendo en cuenta el perfil de riesgo de la población. Método: veinticinco pacientes que participan en el PES con indicación de ITB, con edad promedio de 66,6 años (52-83), todos frecuentando el PES por al menos seis meses. Se midió la presión sistólica en el brazo y la presión arterial sistólica en los tobillos antes y después de la finalización del PES en cicloergómetro de miembros inferiores o una cinta de correr. El ITB se calculó para cada pierna con la utilización del valor más alto como numerador y el valor más alto encontrado en la medida braquial como denominador. Resultados: después del examen, los resultados fueron variados para aumentar, disminuir y mantener los valores de ITB independientemente de la edad, el sexo y las enfermedades presentes en los pacientes estudiados. Conclusión: ningún paciente tuvo ITB $\leq 0,90$, tanto antes como después del ejercicio. El ejercicio demostró ser beneficioso para la irrigación d de las extremidades inferiores.

Palabras clave: índice tobillo braquial, terapia por ejercicio, esfuerzo físico. 


\section{INTRODUÇÃO}

O índice tornozelo-braço (ITB) é o primeiro, o mais simples e o mais importante teste não invasivo para detecção de doença arterial obstrutiva periférica (DAOP). Tem como objetivo medir a integridade da circulação arterial dos membros inferiores. Um valor anormal de ITB $(\leq 0,9)$ apresenta sensibilidade de $90 \%$ e especificidade de $98 \%$ para a detecção de DAOP moderada a grave na angiografia1,2. A medida pode ser realizada em repouso ou após exercício ${ }^{3-7}$. A DAOP dos membros inferiores, tanto sintomática como assintomática, está associada à presença de doença arterial obstrutiva em outros leitos vasculares (coronários, cerebrais, carotídeos) e, por conseguinte, a maior risco de eventos cardiovasculares fatais e não fatais ${ }^{8-14}$. Torna-se fundamental, portanto, que a detecção da DAOP seja realizada precocemente de modo que medidas preventivas possam ser iniciadas e o risco, reduzido. A maior parte dos portadores de DAOP, no entanto, são assintomáticos e as evidências demonstram que apenas questionar a respeito de claudicação intermitente arterial e palpar os pulsos periféricos não é suficiente para a detecção de DAOP dos membros inferiores $^{15,16}$. De modo geral, os principais fatores de risco para doença arterial coronária são também os fatores de risco mais importantes para DAOP. As principais indicações do ITB levando-se em conta o perfil de risco da população são: idade $\leq 65$ anos; idade $\leq 50$ anos com antecedente de diabetes mellitus; idade $\leq 50$ anos com antecedente tabagismo; antecedente de DAOP em outro território, como acidente vascular cerebral, doença arterial coronária, doença oclusiva de carótidas; história familial precoce de doença arterial oclusiva em qualquer território (parentes de $1^{\circ}$ grau, homens idade $\leq 55$ anos e mulheres $\leq 65$ anos); queixas que sugerem claudicação intermitente arterial de membros inferiores; alterações ao exame clínico dos membros inferiores que sugerem DAOP; hipertensão arterial sistêmica; hipercolesterolemia; hiperhomocisteinemia; níveis elevados de Proteína C Reativa ultra-sensível.

Para prescrever exercícios, no programa de exercício supervisionado (PES), devem ser considerados o estado de saúde individual, a presença de fatores de risco, as características comportamentais, os objetivos e as preferências individuais. Fazem parte da prescrição de exercícios componentes essenciais, como o tipo, a duração, a intensidade, a frequência semanal e a progressão das atividades. Os princípios gerais para prescrição de exercícios são aplicados a pessoas com ou sem doenças crônicas, mas algumas condições são diferenciadas na programação, para obter maiores benefícios e evitar complicações ${ }^{17,18}$. A maioria desses programas utiliza uma combinação de tipos de atividades (aeróbicas, exercícios de resistência muscular), em três ou mais sessões semanais, com duração de pelo menos 30 minutos por sessão. Os programas buscam reduzir os sintomas da doença e promover maior independência na realização das atividades diárias ${ }^{19}$. Também podem fazer parte da prescrição exercícios de força e flexibilidade, que são fundamentais para o meIhor desempenho das atividades da vida diária, possibilitando mais autonomia aos pacientes ${ }^{20}$

O ITB pós-esforço pode apresentar duas situações diferentes. Em indivíduos normais ocorre manutenção ou elevação da curva pressórica, demonstrando maior fluxo arterial, e a queda dos níveis pressóricos acontece em indivíduos com diminuição da luz arterial ${ }^{21}$.

Desta forma objetivamos a avaliação e a correlação do ITB com a realização do PES, em indivíduos que apresentam as principais indicações para o exame, levando-se em conta o perfil de risco da população.

\section{MÉTODOS}

Foram escolhidos, de forma randômica, vinte e cinco pacientes participantes do PES na Clínica de Medicina do Exercício Clinimex, localizada na cidade do Rio de Janeiro, RJ, Brasil, todos os pacientes assinaram o Termo de Consentimento Livre e Esclarecido, sendo dezenove homens e seis mulheres, portadores e não portadores de patologias do sistema cardiovascular, porém todos possuíam indicação de serem submetidos ao ITB, e permitiram a realização do exame após o esclarecimento do protocolo a ser realizado e assinarem o Termo de Consentimento Livre e Esclarecido. $O$ trabalho, em nenhum momento interferiu na rotina do PES dos pacientes avaliados. A idade média dos pacientes foi de 66,6 anos $(52-83)$, dezesseis eram portadores de doença arterial coronária (DAC), quinze apresentavam dislipidemia, sete relatavam hipertensão arterial sistêmica (HAS), três com obesidade, dois diabéticos, dois com alterações respiratórias e um saudável. Porém alguns pacientes possuíam mais de uma patologia. Todos faziam uso de medicamentos para suas doenças de base e frequentavam o PES no mínimo há seis meses e relatavam melhor condição de vida após o início do programa de exercício.

Foi medida a maior pressão sistólica no membro superior de forma usual, com esfigmomanômetro (Heine Gamma 3.0 - Alemanha), colocando o manguito no braço do paciente localizando, por meio da palpação, o pulso da artéria braquial, na fossa antecubital, anotado o primeiro som audível ao se desinflar o manguito com auxílio do estetoscópio. A desinflação foi realizada lentamente, de modo que se anote o valor mais exato possível, evitando-se valores aproximados. Foram realizadas pelo menos duas medidas, registrando-se o maior valor. É importante, porém, que a pressão sistólica seja aferida nos dois braços, para evitar níveis sistólicos falsamente reduzidos em um dos braços em virtude de estenose da artéria subclávia, o que não é raro em pacientes portadores de DAOP.

Medimos a pressão sistólica nos tornozelos, utilizando o doppler vascular com transdutor de 5 a $8 \mathrm{MHz}$ com aplicação de gel Sonic ${ }^{\circledR}$ sobre a região analisada, e o mesmo modelo de esfigmomanômetro usado no membro superior, colocando o manguito cerca de três centímetros acima do maléolo. Após palpação dos pulsos pedioso e tibial posterior, procede-se à medida da pressão sistólica sobre a artéria tibial posterior, localizada na goteira retro maleolar medial, em cada membro. É conveniente lembrar que cerca de 10\% da população normal não apresenta pulso pedioso palpável, desta forma, optamos por utilizar o valor encontrado na artéria tibial posterior.

A aferição do ITB foi realizada antes e após a realização do PES, sendo que nas duas situações o paciente estava em posição supina. A aferição antes do PES ocorreu após o paciente ter sido examinado pelo médico assistente, de acordo com a rotina da Clínica; e a aferição após o PES, ocorreu imediatamente após a realização do exercício aeróbico, entre 20 à 30 minutos, programado para cada paciente, de acordo com o programa de exercício individualizado pela clínica e tendo o paciente realizado suas atividades em ciclo ergômetro de membros inferiores ou esteira rolante.

O ITB foi calculado para cada membro inferior, como numerador, o maior valor encontrado em cada membro inferior, e utilizando como denominador o maior valor encontrado nas medidas braquiais.

$$
\mathrm{ITB}=\frac{\text { Maior pressão do tornozelo (tibial posterior) }}{\text { Maior pressão do braço (direito ou esquerdo) }}
$$

A interpretação dos resultados obtidos no ITB foi quantificada da seguinte forma:

valor do ITB > 1,30 artérias não compressíveis; entre 0,91 - 1,30

Normal; entre 0,41 - 0,90 DAOP leve a moderada; e valor do $\mathrm{ITB} \leq 0,40$ DAOP grave. 


\section{RESULTADOS}

Após a realização do exame obtivemos resultados (tabela 1) variados com aumento, diminuição e manutenção dos valores do ITB independente da idade, sexo e patologias presentes nos pacientes analisados.

Pacientes com idade mais avançada obtiveram ITB com índices próximos da normalidade em relação a pacientes mais jovens, como nos casos onze e dezesseis, em comparação aos casos oito e vinte e três, respectivamente.

Dos 16 pacientes portadores de DAC seis (37,5\%) apresentaram diminuição do ITB no pós-esforço, enquanto sete $(43,8 \%)$ obtiveram aumento do índice e três (18,8\%) mantiveram os valores.

Dos 15 pacientes portadores de dislipidemia, seis (40\%) apresentaram diminuição do ITB no pós-esforço, enquanto sete $(46,6 \%)$ obtiveram aumento do índice e dois (13,3\%) mantiveram os valores (tabela 1).

Tabela 1. Resultados obtidos.

\begin{tabular}{|c|c|c|c|c|c|c|c|}
\hline PAC & Sexo & Idade & ITB D pré & ITB E pré & ITB D pós & ITB E pós & $\mathbf{R}$ \\
\hline 1 & $\mathrm{~F}$ & 61 & 1.09 & 1.0 & 1.15 & 1.0 & $\uparrow$ \\
\hline 2 & $M$ & 61 & 1.0 & 1.0 & 1.0 & 1.0 & $=$ \\
\hline 3 & $M$ & 81 & 1.16 & 1.01 & 1.16 & 1.5 & $\uparrow$ \\
\hline 4 & M & 61 & 1.0 & 1.0 & 1.0 & 1.0 & $=$ \\
\hline 5 & M & 74 & 1.15 & 1.15 & 1.18 & 1.18 & $\uparrow$ \\
\hline 6 & M & 52 & 1.28 & 1.0 & 1.33 & 1.16 & $\uparrow$ \\
\hline 7 & $\mathrm{~F}$ & 73 & 1.25 & 1.41 & 1.07 & 1.23 & $\downarrow$ \\
\hline 8 & M & 68 & 1.54 & 1.45 & 1.12 & 1.12 & $\downarrow$ \\
\hline 9 & M & 67 & 1.2 & 1.06 & 1.11 & 0.95 & $\downarrow$ \\
\hline 10 & M & 72 & 1.12 & 1.06 & 1.0 & 1.0 & $\downarrow$ \\
\hline 11 & $M$ & 82 & 1.0 & 1.0 & 1.0 & 1.0 & $=$ \\
\hline 12 & M & 64 & 1.0 & 1.07 & 1.07 & 1.07 & $=$ \\
\hline 13 & $\mathrm{M}$ & 61 & 1.07 & 1.07 & 1.14 & 1.14 & $\uparrow$ \\
\hline 14 & M & 65 & 1.27 & 1.27 & 1.23 & 1.23 & $\downarrow$ \\
\hline 15 & M & 63 & 1.07 & 1.07 & 1.06 & 1.06 & $\downarrow$ \\
\hline 16 & $\mathrm{~F}$ & 83 & 1.08 & 1.08 & 1.0 & 1.0 & $\downarrow$ \\
\hline 17 & $M$ & 73 & 1.16 & 1.33 & 1.14 & 1.21 & $\downarrow$ \\
\hline 18 & $\mathrm{~F}$ & 63 & 1.0 & 1.0 & 1.0 & 1.0 & $=$ \\
\hline 19 & $M$ & 69 & 1.14 & 1.14 & 1.06 & 1.06 & $\downarrow$ \\
\hline 20 & $M$ & 72 & 1.27 & 1.36 & 1.15 & 1.23 & $\downarrow$ \\
\hline 21 & $\mathrm{~F}$ & 69 & 1.25 & 1.26 & 1.12 & 1.12 & $\downarrow$ \\
\hline 22 & $M$ & 59 & 1.16 & 1.0 & 1.23 & 1.15 & $\uparrow$ \\
\hline 23 & $\mathrm{M}$ & 56 & 1.27 & 1.27 & 1.36 & 1.36 & $\uparrow$ \\
\hline 24 & $M$ & 56 & 0.92 & 0.92 & 1.0 & 1.0 & $\uparrow$ \\
\hline 25 & $F$ & 62 & 1.0 & 1.0 & 1.0 & 1.0 & $=$ \\
\hline
\end{tabular}

$P A C=$ pacientes; ITB D pré-índice=tornozelo braço direito pré-esforço; ITB E pré-índice= tornozelo braço esquerdo pré-esforço; ITB D pós-índice=tornozelo braço direito pós- esforço, ITB E pós-índice=tornozelo braço esquerdo pós- esforço; $R=$ resultado; $\uparrow$ = aumento do ITB; $\downarrow$ = diminuição do ITB; = - manutençăo do ITB.

\section{DISCUSSÃO}

Nos pacientes com DAOP sintomática, ITB também tem se mostrado útil como marcador de risco para o desenvolvimento de dor isquêmica em repouso e ulcerações isquêmicas. Em um estudo prospectivo que avaliou 1.244 homens com claudicação intermitente arterial de membros inferiores durante um período de 15 anos, observou-se declínio de 0,014 por ano no ITB, estando este fato diretamente relacionado à piora funcional e ao desenvolvimento de dor e surgimento de úlceras isquêmicas ${ }^{22}$.

O ITB é um preditor independente de eventos cardiovasculares subseqüentes. Em uma avaliação realizada em 5.714 participantes do Cardiovascular Health Study, a maior taxa de mortalidade, após seguimento de seis anos, foi observada nos pacientes que apresentavam doença cardiovascular (DCV) associada a ITB $\leq 0,9$ (32,3\%) e a menor mortalidade foi observada no grupo que não apresentava nenhuma das duas condições $(8,7 \%, p<0,01)$. A mortalidade observada no grupo que não apresentava DCV, mas apresentava ITB $\leq 0,9$, foi consideravelmente elevada $(25,4 \%)^{10}$.

O ITB também foi utilizado para rastrear DAOP e avaliar risco em 1.537 participantes do Estudo SHEP (em inglês Systolic Hypertension and Eldely Program). Os resultados demonstraram que ITB $\leq 0,9$ esteve relacionado a um aumento de dois a três vezes no risco de mortalidade global e de mortalidade cardiovascular em idosos com hipertensão sistólica isolada. Neste estudo, foi possível detectar 19,7\% de pacientes com DAOP subclínica ${ }^{23}$

Em um estudo escocês que avaliou 1.592 indivíduos com idade entre 55 e 74 anos, após cinco anos de seguimento, aqueles com ITB basal $\leq 0,9$ apresentaram aumento de 38\% no risco de infarto do miocárdio não fatal $(\mathrm{RR}=1,38, \mathrm{IC} 95 \%=0,88-2,16)$, de $98 \%$ no risco de acidente vascular cerebral (AVC) (RR=1,98, IC95\%=1,05-3,77), de 85\% na mortalidade cardiovascular $(1,85, \mathrm{IC} 95 \%=1,15-2,97)$ e de $58 \%$ na mortalidade por todas as causas (1,58, IC95\%=1,14-2,18), após ajustar os resultados para idade, sexo, presença de doença arterial coronária (DAC) e diabetes. Foi interessante observar que o valor preditivo para eventos adversos aumentou de forma significativa ao se combinar o valor do ITB aos outros fatores clássicos de risco cardiovascular. O valor preditivo positivo em fumantes hipertensos, por exemplo, aumentou de $25 \%$ para $43,8 \%$ nos pacientes que apresentaram ITB $\leq 0,9^{10}$. Embora seja um método utilizado para detectar DAOP subclínica, o ITB não detectará estenoses mínimas. É necessária, portanto, a presença de DAOP moderadamente avançada para que o valor do ITB se encontre diminuído. Desta forma, para se tornar custo-efetivo a medida do ITB, como forma de detecção precoce de DAOP, deve ser direcionada para populações de alto risco para o desenvolvimento da doença. Estudos realizados com esse objetivo demonstraram taxas de detecção de novos casos de DAOP de até 30\% nas populações de alto risco. No programa USA PARTNERS, que avaliou 6.417 pacientes com idade $>$ 70 anos ou com idade entre 50 e 69 anos, desde que fossem fumantes e/ou diabéticos, dois importantes fatores de risco para DAOP, a taxa de detecção de DAOP foi de 29\%, dos quais 45\% representavam novos casos da doença ${ }^{24}$

No trabalho realizado observamos que existiam pacientes com diagnóstico de dislipidemia, fator que leva a diminuição da luz arterial em todos os leitos vasculares. Observamos, também, que existia a associação entre dislipidemia e DAC (12 pacientes - 48\%), e que estes possuíam diminuição do ITB pós-esforço, comprovando a diminuição de irrigação nos tecidos, porém nenhum deles apresentou ITB $\leq 0,9$. Apesar de todos os pacientes analisados serem portadores de algum grau de aterosclerose, seja pelas doenças de base ou simplesmente pela idade (a partir da sexta década), foi demonstrando que a atividade física regular mantém os índices pressóricos em níveis satisfatórios.

O ITB tem sido o método preferido para detectar DAOP subclínica de membros inferiores por se tratar de um método não invasivo, de baixo custo, de fácil realização e capaz de detectar a DAOP assintomática ${ }^{25}$. A incorporação dessa ferramenta como parte da avaliação cardiovascular de rotina é altamente recomendável na prática clínica diária dos profissionais que lidam com pacientes de alto risco cardiovascular ${ }^{26}$.

Valores de ITB superiores a 1,3 podem ocorrer na doença aterosclerótica difusa nos vasos com calcificação e rigidez da parede vascular tornando as artérias não compressíveis. Ocorre com mais frequência em pacientes diabéticos, com insuficiência renal crônica, e em idosos e pode levar a resultados falso-negativos. Deve-se suspeitar de artérias não compressíveis ao se observar que o ruído obtido pelo doppler não 
desaparece ao se inflar o manguito, $\geq 250 \mathrm{mmHg}$. Nestes casos, avaliações vasculares mais sofisticadas devem ser indicadas para detectar a presença de DAOP.

\section{CONCLUSÕES}

Nenhum paciente obteve ITB $\leq 0,90$, tanto no pré como no pós-esforço, pois todos apresentam regularidade nas atividades físicas, a melhor terapêutica para DAOP.

O exercício mostrou-se benéfico para a irrigação dos membros inferiores, independente da patologia de base.

\section{AGRADECIMENTOS}

Aos dirigentes, professores e aos membros da equipe multidisciplinar da Clínica de Medicina do Exercício Clinimex, do Rio de Janeiro, RJ, Brasil, pelo apoio e orientação na confecção deste trabalho, bem como a permissão e participação voluntária de seus frequentadores.

Todos os autores declararam não haver qualquer potencial conflito de interesses referente a este artigo.

\section{REFERÊNCIAS}

1. Yao ST, Hobbs JT, Irvine WT. Ankle systolic pressure measurements in arterial disease affecting the lower extremities. Br J Surg. 1969;56(9):676-9.

2. Ouriel $K$, McDonnell $A E$, Metz CE, Zarins CK. Critical evaluation of stress testing in the diagnosis of peripheral vascular disease. Surgery. 1982;91(6):686-93.

3. Belch JJ, Topol EJ, Agnelli G, Bertrand M, Califf RM, Clement DL, et al. for Critical issues in peripheral arterial disease detection and management: a call to action. Arch Intern Med. 2003;163:884-92.

4. Jaff MR. Diagnosis of peripheral arterial disease: utility of the vascular laboratory. Clin Cornerstone. 2002;4(5):16-25.

5. Schmieder FA, Comerota AJ. Intermittent claudication: magnitude of the problem, patient evaluation, and therapeutic strategies. Am J Cardiol. 2001;87(12A):D3-13.

6. Brito CJ. Cirurgia vascular: cirurgia endovascular, angiologia. Rio de Janeiro: Revinter; 2002.

7. Thomaz JB, Herdy CD. Fundamentos de cirurgia vascular e angiologia. São Paulo: Fundo Editorial Byk; 1997.

8. Newman AB, Siscovick DS, Manolio TA, Polak J, Fried LP, Borhani NO, et al. Ankle-brachial index as a marker of atherosclerosis in the cardiovascular health study. Circulation. 1993;88(3):837-45.

9. Leng GC, Fowkes FGR, Lee AJ, Dunbar J, Housley E, Ruckley CV. Use of anke brachial index to predict cardiovascular events and death: a cohort study. BMJ. 1996;313(70701):1440-4.

10. Newman AB, Shemanski L, Manolio TA, Cushman M, Mittelmark M, Polak JF, et al. Ankle-brachial index as a predictor of cardiovascular diseaseand mortality in the cardiovascular health study. Arteriocler Thromb Vasc Biol. 1999;19:538-45.

11. Garcia LA. Epidemiology and pathophysiology of lower extremity peripheral arterial disease. J Endovasc Ther. 2006;13(Suppl 2):3-9.

12. Hilleman DE. Management of peripheral arterial disease. Am J Health Syst Pharm. 1998;55(19 Suppl 1):21-7.

13. Lamina C, Meisinger C, Heid IM, Rantner B, Döring A, Löwel H, et al. Ankle-brachial index and peripheral arterial disease. Gesundheitswesen. 2005;67(Suppl 1):57-61.

14. Norman PE, Eikelboom JW, Hankey GJ. Peripheral arterial disease: prognostic significance and prevention of atherothrombotic complications. Med J Aust. 2004;181(3):150-4.

15. Criqui MH, Coughçlin SS, Fronek A. Noninvasively diagnosed peripheral arterila disease as a predictor of mortality: results from a prospective study. Circulation. 1985; 72(4):768-73

16. Lombardi G, Polotti R, Polizzi N, Inzoli MR. Prevalence of asymptomatic peripheral vascular disease in a group of patients older than 50. J Am Geriatr Soc. 1986;34(7):551-2.

17. American College of Sports Medicine. ACMS's guidelines for exercise testing and prescription. 6 th ed. Philadelphia: Lippincott Williams \& Wilkins; 2000.

18. Yazbek Júnior P, Battistella LR. Condicionamento físico do atleta ao transplantado. São Paulo: Savier; 1994 19. Gardner AW, Poehlman ET. Exercise rehabilitation programs for the treatment of claudication pain. JAMA. 1995;274(12):975-80

20. Gardner, A. W. Peripheral arterial disease. In: American College of Sports Medicine. ACSM's exercise management for persons with chronic disease and disabilities. Champaign- IL: Human Kinetics; 1997. p. 64-8.

21. Maffei FH. Doenças vasculares periférica. 3a ed. Rio de Janeiro: Medsi; 2002.

22. Aquino R, Johnides C, Makaroun M, Whittle JC, MulukVS, Kelley ME, et al. Natural history of claudication. Long-term serial follow-up study of 1244 cludicants. J Vasc Surg. 2001;34(6):962-70.

23. Newman AB, Tyrrell KS, Kuller LH. Mortality over four years in SHEP participants with a low ankle-arm index. J Am Geriatr Soc. 1997;45(12):1472-8.

24. Hirsch AT, Criqui MH, Treat-Jacobson D, Regensteiner JG, Creager MA, Olin JW, et al. Peripheral arterial disease detection, awareness, and treatment in primary cale. JAMA. 2001;286(11):1317-24.

25. McDermott MM, Fried L, Simonsick E, Ling S, Guralnik JM. et al. Asymptomatic peripheral arterial disease is independently associated with impaired lower extremity functioning. The women's health ands aging study. Circulation. 2000;101(9):1007-12.

26. Kaiser V, Hooi JD, Stoffers HE, Van der Laan JR. NHG-Standaard perifeer arterieel vaatlijden. In: Geijer RMM, Burgers JS, Van der Laan JR, Wiersma TJ, Rosmalen CFH, Thomas S. NHG practice guidelines for the general practitioner. Part I. Maarssen: Elsevier/Bunge; 1999. 